\title{
SIGMA-COMPACT LOCALLY CONVEX METRIC LINEAR SPACES UNIVERSAL FOR COMPACTA ARE HOMEOMORPHIC
}

\author{
T. DOBROWOLSKI AND J. MOGILSKI
}

\begin{abstract}
It is proved that every $\sigma$-compact locally convex metric linear space containing a topological copy of the Hilbert cube $Q$ is homeomorphic to $\Sigma$ the linear span of the Hilbert cube $Q$, in the Hilbert space $l_{2}$.
\end{abstract}

Let us fix the following representation of the Hilbert cube $Q$ in the Hilbert space $l_{2}$ :

$$
Q=\left\{\left(x_{n}\right) \in l_{2}:\left|x_{n}\right| \leq n^{-2} \text { for every } n\right\} .
$$

Consider the space $\Sigma=\operatorname{span}(Q) \subset l_{2}$. We note that $\Sigma$ is a $\sigma$-compact linear dense subspace of $l_{2}$. The purpose of this note is to prove the following fact:

THEOREM. Every $\sigma$-compact locally convex metric linear space $E$ containing a topological copy of the Hilbert cube $Q$ is homeomorphic to $\Sigma$. Moreover, if $\tilde{E}$ is the completion of $E$, then the pairs $(\tilde{E}, E)$ and $\left(l_{2}, \Sigma\right)$ are homeomorphic.

The Theorem resolves the outstanding problem LS3 in [4] posed by Anderson in connection with the fact that the assertion of the Theorem was known to be true under the additional assumption that the copy of the Hilbert cube above was a convex set (see: Anderson [1], Bessaga-Petczyński [2] and Toruńczyk [5]). Let us recall that the classical theorem of Keller-Klee [3, p. 100] states that every metrizable infinite-dimensional compact convex subset $K$ of a locally convex space is homeomorphic to $Q$. We complete the paper with an example of a $\sigma$-compact dense linear subspace of $l_{2}$ which contains a topological copy of $Q$ and does not contain any infinite-dimensional compact convex subset. This shows that the Theorem cannot be reduced to the above-mentioned result of $[1,2,5]$.

The proof of the Theorem involves the technique of absorbing sets. We will use the approach of West [8] and Torunczyk [6] instead of the original approaches of Anderson (cap-set technique [1]) and Bessaga-Petczyński (skeleton technique [2]). According to $[8,6]$ a $\sigma$-compact set $M$ is an absorbing set (for compacta) in a space $X$ homeomorphic to $l_{2}$ if the following condition is satisfied:

(abs) every map $f: Q \rightarrow X$ such that $f \mid B$ is an embedding of a closed set $B \subset Q$ into $M \subset X$ can be approximated by embeddings $g: Q \rightarrow M$ with $g|B=f| B$.

The space $\Sigma$ may serve as an example of an absorbing set in $l_{2}$ (see [3, p. 275]). We intend to show that the space $E$ is also an absorbing set in the completion $\tilde{E}$ homeomorphic, by the Kadec-Anderson theorem [3, p. 189], to $l_{2}$. Then the assertion of our Theorem will follow from the theorem on equivalence of absorbing sets $[8,6]$ stating that: if $M_{i}$ is an absorbing set in $X_{i}(i=1,2)$, then the pairs $\left(X_{1}, M_{1}\right)$ and $\left(X_{2}, M_{2}\right)$ are homeomorphic.

Received by the editors July 6,1981 .

1980 Mathematics Subject Classification. Primary 57N17, 57N20.

$K e y$ words and phrases. $\sigma$-compact linear span, linear space, topological embedding.

(c) 1982 American Mathematical Society 0002-9939/82/0000-0396/802.25 
The verification of the condition (abs) makes use of the Proposition below. We shall assume that $|\cdot|$ is an $F$-norm on a locally convex space $E$, i.e., a function $|\cdot|: E \rightarrow[0, \infty)$ such that $(x, y) \rightarrow|x-y|$ defines a compatible metric on $E$. We shall additionally require that each ball

$$
N(\eta)=\{x \in E:|x|<\eta\}
$$

is convex. We shall often write, for $\varphi, \psi: Y \rightarrow E$

$$
|\varphi-\psi|=\sup \{|\varphi(y)-\psi(y)|: y \in Y\}
$$

The symbol $H(Y)$ denotes the space of all self-homeomorphisms of $Y$.

Proposition. Let $(E,|\cdot|)$ be an infinite-dimensional locally convex space and let $B$ and $A$ be disjoint compacta of $E$. Then, given $a$ map $f: B \rightarrow E$ and $\epsilon>0$, there exists a homeomorphism $h \in H(E)$ satisfying

(i) $|h-\mathrm{id}|<\left|f-\mathrm{id}_{B}\right|+\epsilon$;

(ii) $h \mid A=\mathrm{id}_{A}$;

(iii) $|h| B-f \mid<\epsilon$.

LEMMA 1. Assuming that $\operatorname{span}(B \cup f(B))$ is finite-dimensional and that $f$ is an embedding with $f(B) \cap A=0$, there exists $h \in H(E)$ extending $f$ and satisfying the conditions (i) and (ii) of the Proposition.

LEMMA 2. Given a positive $\delta$, there exists a map

$$
\text { u: } E \times[0,1) \cup B \times\{1\} \rightarrow E
$$

such that, writing $u_{t}=u(\cdot, t)$, we have $u_{1}(B) \cap A=\emptyset, \operatorname{span}\left(u_{1}(B)\right)$ is finitedimensional and, for $t<1$,

(iv) $u_{t} \in H(E)$ with $u_{0}=\mathrm{id}$;

(v) $\left|u_{t}-\mathrm{id}\right|<\delta$;

(vi) $u_{t} \mid A=\mathrm{id}_{A}$.

ProOf OF LEMMA 1. Pick a positive $\eta$ with $\eta<\operatorname{dist}(A, B \cup f(B))$ and $\eta<\epsilon$. The compactness of $A$ implies that there is a finite-dimensional linear subspace $E^{\prime}$ of $E$ satisfying

$$
A \subset E^{\prime}+N(\eta / 16)
$$

and such that $E_{1}=\operatorname{span}(B \cup f(B)) \subset E^{\prime}$ and $\operatorname{dim}\left(E^{\prime}\right) \geq 2 \cdot \operatorname{dim}\left(E_{1}\right)+3$. Consider the set $N(\eta / 2) \backslash\left(E^{\prime}+N(\eta / 4)\right)=C$. If $C \neq \emptyset$, pick a point $x_{0} \notin E^{\prime}+N(\eta / 4)$ to have

$$
\left|x_{0}\right|<\eta / 2 \text {. }
$$

Otherwise, there exists a point $x_{0} \in E \backslash E^{\prime}$ such that

$$
\left|s x_{0}\right|<\eta / 2 \text { for every } s \geq 0 \text {. }
$$

In either case, let $E_{0}=\operatorname{span}\left(E^{\prime},\left\{x_{0}\right\}\right)$. By a theorem of Michael [3, p. 85], there exists a continuous right inverse $\varphi: E / E_{0} \rightarrow E$ for the quotient map $\kappa: E \rightarrow E / E_{0}$; by the local convexity of $E$ we may additionally require that

$$
|\varphi(\kappa(x))| \leq 2|x| \text { for all } x \in E .
$$


Writing $r(x)=x-\varphi(\kappa(x))$, by (1) and (3), we have

$$
r(A) \subset A+N(\eta / 8) \text {. }
$$

The formula

$$
\alpha_{t}(b)=\left\{\begin{array}{l}
b+t x_{0}, \quad t \in[0,1] \\
(2-t) \cdot\left(b+x_{0}\right)+(t-1) \cdot\left(f(b)+x_{0}\right), \quad t \in[1,2], \\
f(b)+(3-t) x_{0}, \quad t \in[2,3]
\end{array}\right.
$$

defines a homotopy $\alpha$ joining id ${ }_{B}$ with $f$ in $E_{0}$. By (2), we have

$$
\left|\alpha_{t}(b)-b\right|<\eta / 2+|f(b)-b|+\eta / 2<\epsilon+|f(b)-b|
$$

for every $b \in B$. Moreover, by (4), (2) or ( $\left.2^{\prime}\right)$ we get

$$
\alpha(b \times[0,3]) \cap r(A)=\emptyset .
$$

(In the case where $C=\emptyset$, in the formula describing $\alpha$, we put instead of $x_{0}$, the point $s x_{0}$ in order to have $\left(s x_{0}+E^{\prime}\right) \cap r(A)=0$.) The space $E_{0}$ has the estimated extension homeomorphism property for compacta $K$ with $\operatorname{dim}(\operatorname{span}(K)) \leq$ $\operatorname{dim}\left(E_{1}\right)+1$ (we apply, for instance, the "graph-trick" of Klee [3, p. 62]; see also $\left[4\right.$, p. 11]. Consequently, there exists a homeomorphism $\bar{h} \in H\left(E_{0}\right)$ with $\bar{h} \mid B=f$ satisfying, by (5),

$$
|\bar{h}-\mathrm{id}|<\epsilon+\left|f-\mathrm{id}_{B}\right|
$$

and such that, by (6), $\bar{h} \mid r(A)=\mathrm{id}_{r(A)}$. Finally, we put $h(x)=\varphi(\kappa(x))+\bar{h}(r(x))$ for $x \in E$.

Proof of LEMMA 2. Assume that $\delta<\operatorname{dist}(A, B)$ and consider a finitedimensional linear space $E_{0} \subset E$ with

$$
A \cup B \subset E_{0}+N(\delta / 4) \text {. }
$$

Let $\varphi: E / E_{0} \rightarrow E$ be a map of the proof of Lemma 1 (i.e. $\varphi$ satisfies the condition (3)). Write $h_{0}(x)=(\kappa(x), r(x))$ for the homeomorphism of $E$ onto $E / E_{0} \times E_{0}$ with $r(x)=x-\varphi(\kappa(x))$. Let $|\cdot|$ be the quotient $F$-norm on $E / E_{0}=Y$. By (7), we have $r(A) \cap r(B)=\emptyset$. Let $\omega: E_{0} \rightarrow[0, \delta / 4]$ be a map with

$$
h_{0}(B) \subset\left\{(y, e) \in Y \times E_{0}:|y|<\omega(e)\right\} \subset h_{0}(E \backslash A) .
$$

Let $\left\{0<\alpha_{t} \leq 1\right\}, 0 \leq t<1$, be a homotopy of $[0, \infty]$ with $\alpha_{0} \equiv 1, \alpha_{t}$ is monotone and $\alpha_{t} \mid[1, \infty]=1$ for each $t$ and such that for each $s<1, \lim _{t \rightarrow 1} \alpha_{t}(s)=0$. Define an isotopy $\left\{g_{t}\right\}, 0 \leq t<1$, of $Y \times E_{0}$ by the formula

$$
g_{t}(y, e)=\left\{\begin{array}{l}
\left(\alpha_{t}(|y| / \omega(e)) \cdot y, e\right), \quad \text { if } y \neq 0, \\
(0, e), \quad \text { if } y=0 .
\end{array}\right.
$$

Then the desired map $u$ may be defined by $u(x, t)=h_{0}^{-1} g_{t} h_{0}(x)$ for $0 \leq t<1$, and $u(b, 1)=r(b)$ for $b \in B$.

Proof of Proposition. (1) Assume $\operatorname{span}(B \cup f(B))=E_{1}$ is finitedimensional. One can easily approximate $f$ by maps with ranges disjoint from $A$. Thus, we will require that $f(B) \cap A=\emptyset$. Since $B$ is finite-dimensional, there exists an embedding $w: B \rightarrow E$ with $|w(b)|<\delta$ and such that $E_{2}=\operatorname{span}(w(B))$ is finite-dimensional with $E_{2} \cap E_{1}=\{0\}$. Writing $v=f+w$, we see that $v$ is an 
embedding satisfying

$$
\left|v-\operatorname{id}_{B}\right|<|f-\mathrm{id}|+\delta \text { and }|v-f|<\delta .
$$

If $\delta$ is sufficiently small, we also get

$$
v(B) \cap A=\emptyset .
$$

Assuming $\delta<\epsilon$ and applying Lemma 1, we extend the embedding $v$ to a required homeomorphism $h$.

(2) The general case. Let $\bar{f}: E \rightarrow E$ be a map with $\vec{f} \mid B=f$ (we use the AR-property of $E$ ). Pick $\delta$ with $0<\delta<\epsilon / 8$ and such that

$$
b \in B, \quad|x-b|<\delta \quad \text { implies }|\bar{f}(x)-f(b)|<\epsilon / 4 .
$$

With this $\delta$, consider a homotopy $u_{t}$ of Lemma 2 and write $B^{\prime}=u_{1}(B)$. Let us approximate $\bar{f} \mid B^{\prime}$ by $f^{\prime}: B^{\prime} \rightarrow E$ such that

$$
|\bar{f}| B^{\prime}-f^{\prime} \mid<\epsilon / 4 \text { and } \operatorname{dim}\left(\operatorname{span}\left(f^{\prime}\left(B^{\prime}\right)\right)\right)<\infty .
$$

Now, observing that the triple $\left(B^{\prime}, A, f^{\prime}\right)$ satisfies the assumptions of the previous case, we conclude that there exists $h^{\prime} \in H(E)$ satisfying $\left|h^{\prime}\right| B^{\prime}-f^{\prime} \mid<\epsilon / 4$, $\left|h^{\prime}-\mathrm{id}\right|<\left|f^{\prime}-\mathrm{id}_{B^{\prime}}\right|+\epsilon / 4$ and $h^{\prime} \mid A=\mathrm{id}_{A}$. We claim that $h_{t}=h^{\prime} \circ u_{t}$ may serve as a required homeomorphism, provided that $t \neq 1$ is sufficiently close to 1 .

First observe that for $b^{\prime}=u_{1}(b) \in B^{\prime}$, we can estimate, using (11), (v) and (10),

$$
\begin{aligned}
\left|f^{\prime}\left(b^{\prime}\right)-b^{\prime}\right| \leq & \left|f^{\prime}\left(b^{\prime}\right)-\bar{f}\left(b^{\prime}\right)\right|+\left|\bar{f}\left(u_{1}(b)\right)-f(b)\right|+|f(b)-b| \\
& +\left|b-u_{1}(b)\right| \leq \epsilon / 4+\epsilon / 4+\left|f-\operatorname{id}_{B}\right|+\epsilon / 8 .
\end{aligned}
$$

Consequently, by (v), for all $t<1$, we have

$$
\begin{aligned}
\left|h_{t}-\mathrm{id}\right| & \leq\left|h^{\prime} \circ u_{t}-u_{t}\right|+\left|u_{t}-\mathrm{id}\right| \leq\left|h^{\prime}-\mathrm{id}\right|+\epsilon / 8 \\
& \leq 5 \epsilon / 8+\left|f-\mathrm{id}_{B}\right|+\epsilon / 4+\epsilon / 8 \leq\left|f-\mathrm{id}_{B}\right|+\epsilon .
\end{aligned}
$$

Now, we take $t_{0} \in(0,1)$ such that for every $t \geq t_{0}$ we have

$$
\left|h^{\prime}\left(u_{t}(b)\right)-h^{\prime}\left(u_{1}(b)\right)\right|<\epsilon / 4 \quad \text { for all } b \in B .
$$

Using (12)-(10), for $t \in\left[t_{0}, 1\right)$, we have

$$
\begin{aligned}
\left|h_{t}(b)-f(b)\right|= & \left|h^{\prime} u_{t}(b)-f(b)\right| \leq\left|h^{\prime} u_{t}(b)-h^{\prime} u_{1}(b)\right| \\
& +\left|h^{\prime} u_{1}(b)-f^{\prime} u_{1}(b)\right|+\left|f^{\prime}\left(u_{1}(b)\right)-\bar{f}\left(u_{1}(b)\right)\right| \\
& +\left|\bar{f} u_{1}(b)-f(b)\right|<\epsilon / 4+\epsilon / 4+\epsilon / 4+\epsilon / 4 .
\end{aligned}
$$

Finally, since both $h^{\prime}$ and $u_{t}$ are the identity on $A$, the composition $h_{t}=h^{\prime} \circ u_{t}$ has this property also.

PROOF OF THEOREM. By what was said in the Introduction we have to verify the condition (abs) for the pair $(X, M)=(\tilde{E}, E)$. Since the set $\tilde{E} \backslash E$ is locally homotopy negligible in $\tilde{E}$ (see [7, Remark 2.9 and Theorem 2.4]), the map $f: Q \rightarrow$ $\tilde{E}$ can be approximated by maps which are equal to $f$ on $B$ and have ranges in $E$. Using our hypothesis, we may assume that the Hilbert cube is placed in the space $E$; possibly with $Q \cap f(Q)=0$.

Fix a positive $\epsilon$. Consider a tower $\left\{A_{i}\right\}_{i=0}^{\infty}$ consisting of compacta, with $A_{0}=$ $\checkmark$ and $\bigcup_{i=0}^{\infty} A_{i}=Q \backslash B$. We shall inductively construct a sequence of homeomorphisms $\left\{h_{n}\right\}_{n=0}^{\infty} \subset H(E)$ such that, writing $g_{n}=h_{n} \circ h_{n-1} \circ \cdots \circ h_{0}$, the following 
conditions are satisfied:

$$
\left|g_{n}-g_{n-1}\right|<2^{-n+1} \epsilon
$$

and

$$
\begin{gathered}
g_{n}\left|A_{n-1}=g_{n-1}\right| A_{n-1} \quad \text { for } n=1,2, \ldots ; \\
\left|g_{n}\right| B-f|B|<2^{-n-1} \epsilon
\end{gathered}
$$

and

$$
g_{n} \mid f(Q)=\text { id } \quad \text { for } n=0,1,2, \ldots
$$

Let $h_{0}$ be a homeomorphism obtained by the Proposition applied to the quadruple $(Q, f, f(Q), \epsilon / 2)$. Assume $h_{0}, \ldots, h_{n-1}(n \geq 1)$ are already constructed. To get $h_{n}$, apply the Proposition to the quadruple $\left(g_{n-1}(B), f \circ g_{n-1}^{-1} \mid g_{n-1}(B), g_{n-1}\left(A_{n}\right) \cup\right.$ $\left.f(Q), 2^{-n-1} \epsilon\right)$. Then (13) is a consequence of the calculation

$$
\begin{aligned}
\left|g_{n}-g_{n-1}\right| & =\left|h_{n}-\mathrm{id}\right|<\left|f \circ g_{n-1}^{-1}\right| g_{n-1}(B)-\mathrm{id}_{g_{n-1}(B)} \mid+2^{-n-1} \epsilon \\
& \leq|f| B-g_{n-1}|B|+2^{-n-1} \epsilon \leq 2^{-n} \epsilon+2^{-n-1} \epsilon<2^{-n+1} \epsilon .
\end{aligned}
$$

The conditions (14)-(16) also follow.

Now, put $g(q)=\lim g_{n}(q)$ for $q \in Q$. By (13)-(15), $g$ is a continuous map from $Q$ into $E$ such that $g|B=f| B$ and $|g-f|<3 \epsilon$. Finally, by (14) and (16), $g$ is a 1-1 map (and therefore an embedding of $Q$ into $E$ ).

EXAMPLE. Let $i: Q \rightarrow l_{2}$ be an embedding of the Hilbert cube $Q$ into the Hilbert space $l_{2}$ such that the set $Q^{\prime}=i(Q)$ is linearly independent (see [3, p. 193] and the Note below). Let $E=\operatorname{span}\left(Q^{\prime}\right) \subset l_{2}$. Since $E$ can be expressed $E=\bigcup_{n=1}^{\infty} A_{n}$, with $A_{n}=\left\{\sum_{k=1}^{n} t_{k} \cdot q_{k}^{\prime}:\left|t_{k}\right| \leq n, q_{k}^{\prime} \in Q^{\prime}\right\}, E$ is $\sigma$-compact. The space $E$ does not contain any infinite-dimensional compact convex subset.

ProOF. First, we verify that every convex subset $C$ contained in $A_{n}$ has dimension $\leq n$. Let $m$ be the maximal integer such that there is $c=\sum_{k=1}^{m} t_{k} \cdot q_{k}^{\prime} \in$ $C$ with $t_{k} \neq 0$. We claim that $C \subset \operatorname{span}\left(\left\{q_{1}^{\prime}, \ldots, q_{m}^{\prime}\right\}\right)$. If there were $c_{0} \in C$ such that $c_{0}=\sum_{k=1}^{m} s_{k} \cdot q_{k}^{\prime}+\sum_{k=m+1}^{l} s_{k} \cdot q_{k}^{\prime}$ with $s_{k} \neq 0$ for $k=m+1, \ldots, l$, then we would find a suitable $t \in(0,1)$ such that the point $t c+(1-t) c_{0} \in C$ would have all $l$ coordinates different from 0 . This contradicts the maximality of $m$.

Now, assuming that $E$ contains an infinite-dimensional compact convex subset, by a Baire category argument, there is $A_{n_{0}}$ which also contains such a subset. However, by the above argument, this is impossible.

Note. Let $E$ be a locally convex metric space containing an infinite-dimensional compact convex set $C$. Then, $E$ contains a dense $\sigma$-compact linear subspace $E_{0}$ such that: (a) $E_{0}$ contains a topological copy of the Hilbert cube; and (b) $E_{0}$ does not contain any infinite-dimensional compact convex subset. This is a consequence of the fact that there exists an embedding of $Q$ onto a linearly independent subset $C^{\prime}$ of $C$ with $\mathrm{cl}\left(\operatorname{span}\left(C^{\prime}\right)\right) \supset \operatorname{span}(C)$. (To see this, consider $C$ as a subset of $l_{2}$ with $0 \in C$ (use [3, p. 100]) and take an orthogonal set $\left\{e_{i}\right\}_{i=1}^{\infty} \subset C$ which is total for $\operatorname{span}(C)$. The formula $\left(x_{n}\right) \rightarrow \sum_{n=1}^{\infty} \sum_{k=1}^{\infty} x_{n}^{k} \cdot e_{n(k)}$, where $\{n(k): k=1,2, \ldots\}$ is a partition of the integers into countable sets, defines a required embedding.

The authors are grateful to W. Smolenski who pointed out that the space $E$ of the example does not contain any infinite-dimensional compact convex subset. 


\section{REFERENCES}

1. R. D. Anderson, On sigma-compact subsets of infinite-dimensional manifolds, Louisiana State Univ., preprint.

2. C. Bessaga and A. Pelczyński, The estimated extension theorem, homogeneous collections and skeletons, and their application to the topological classification of linear metric spaces and convex sets, Fund. Math. 69 (1970), 153-190.

3. __ Selected topics in infinite-dimensional topology, PWN, Warszawa, 1975.

4. T. A. Chapman, Lectures on Hilbert cube manifolds, CBMS Regional Conf. Ser. Math., no. 28, Amer. Math. Soc., Providence, R.I., 1976.

5. H. Torunczyk, Skeletonized sets in complete metric spaces and homeomorphisms of the Hilbert cube, Bull. Acad. Polon. Sci. Sér. Sci. Math. Astronom. Phys. 18 (1970), 119-126.

6. _ـ , $(G-K)$-absorbing and skeletonized sets in metric spaces, Ph. D. Thesis, Inst. Math., Polish Acad. Sci., Warsaw, 1970.

7. __, Concerning locally homotopy negligible sets and characterizations of $l_{2}$-manifolds, Fund. Math. 101 (1978), 93-110.

8. J. E. West, The ambient homeomorphy of incomplete subspaces of infinite-dimensional Hilbert spaces, Pacific J. Math. 34 (1970), 257-267.

Institute OF MAThematics, WARsaW UNIVERsity, PKIN 00-901 WARSAW, POLAND 